\title{
Deprescribing: is the law on your side?
}

\author{
Nina Barnett, ${ }^{1}$ Orla Kelly ${ }^{2}$
}

\begin{abstract}
${ }^{1}$ Pharmacy Department, Northwick Park Hospital, Harrow, UK ${ }^{2}$ Cantillons Solicitors, Cork, Ireland
\end{abstract}

\section{Correspondence to} Orla Kelly, Healthcare Litigation Solicitor and Pharmacist, Cantillons Solicitors, 38/39 South Mall, Cork, T12 W522, Ireland; orla.kelly@cantillons.com

Received 7 April 2016 Revised 10 May 2016 Accepted 16 May 2016

\section{ABSTRACT}

Deprescribing can feel risky: prescribers need to consider the consequence of stopping a medication medicolegally, particularly where there may be a guideline or accepted practice that suggests its use. This review aims to provide reassurance and encouragement to safely deprescribe. Experience suggests that for many patients the prescribing of multiple medicines is the norm. In the right setting and at the right time, deprescribing provides a real opportunity to minimise side effects and unwanted interactions through patient-centred conversations. How does the prescriber stand if they stop a medication for which there is an evidence-based guideline to use it? This review explores the concepts of clinical negligence and informed patient consent in the context of deprescribing. Using examples from UK case law, the review discusses the legal tests which are applied to establish an action based on clinical negligence and lack of informed consent. It describes the recent changes in law which reinforce the importance of providing information focused on what is material to individual patients. The use of prescribing tools to support safe deprescribing and informed consent are also discussed. When deprescribing is undertaken in partnership with patients, supported by the knowledge, skills and experience of both patient and clinicians and the patient's values and preferences based on clinical skill, judgement and evidence-based medicine, law presents no barriers to deprescribing.

This review considers the meaning of deprescribing; exploring both the theoretical and practical benefits of deprescribing and discusses the legal issues around deprescribing in the context of current practice. Clinicians are encouraged, if not mandated, to follow numerous local and national guidelines which support appropriate, safe and evidence-based prescribing, however most offer little or no guidance around deprescribing. For this reason, deprescribing can feel risky and fear of litigation may lead prescribers to consider the consequence of stopping a medication medicolegally, particularly where there may be a guideline or accepted practice that suggests its use. Arguably the practice of prescribing is inherently risk averse. However, this is not a criticism. As prescribers and pharmacists, we work with patients to make interventions that affect patients' lives for which considered, cautious decision making is required.

Against this background, we consider the following question: is deprescribing exposing clinicians to potential litigation?

This review cites the law in the UK which is based on a common law legal system. While the clinical recommendations to embrace deprescribing are relevant in a pan-European context, the legal observations may vary across different countries and legal codes.

\section{WHY SHOULD WE CONSIDER DEPRESCRIBING?}

In order to understand our current challenge, it is useful to consider the history of prescribing and how this relates to the present day context. While today prescribing is the most common healthcare intervention, this is only true for the last 70 years. Prior to this, few licensed medicines existed. Medication choice and formulation were particular to individual doctors who more often than not did not share their rationale or formulae with colleagues.

In the pre-National Health Service (NHS) era (pre 1948) in the UK, patients self-funded their treatment and thus financial considerations may have been a factor for clinicians in parsimonious prescribing to minimise expenditure for patients. As licensed medicines became available, they were treated as both a scarce and valuable resource. In addition, medication was aimed at managing acute illness and/or symptomatic treatment in the short term and the concept of preventative medicine, where no immediate benefit was seen, was rare.

In the 1950s and 1960s medicines became more widely available and the choice of effective therapies increased. Preventative therapies began to emerge to add to the arsenal of curative and symptomatic therapies already available. With the advent of critical appraisal in the 1970s and 1980s, evidence-based guidelines began to appear. ${ }^{1}$ Variation in prescribing practice began to reduce in the UK and from the 1990s onwards prescribers were strongly encouraged to use guidelines as part of routine prescribing practice. The Cochrane collaboration ${ }^{2}$ and then the National Institute for Health and Care Excellence ${ }^{3}$ as well as many Professional Bodies and Royal Colleges have contributed to the plethora of guidelines now in existence which support evidence-based prescribing. Guidelines ensure that prescribers are aware of which cohort of patients could benefit from taking a particular treatment. However, for the most part, guidelines focus on which patients could benefit from initiation of a medication and not on when to review, reduce or stop it. When initiating a medicine, the options of stopping or reassessing a medicine in due course are rarely discussed.

We are now in an era where the prescribing of multiple medicines is common and experience suggests that for many patients it is becoming the norm. This has both clinical and financial implications for the NHS. While in the 1980s patients were often discharged from hospital with just two or three medications, ${ }^{4}$ discharge prescriptions today commonly contain eight medicines. ${ }^{5}$ One of 
the authors recently learnt of a patient discharged from hospital with 42 medications. Medication review is an important opportunity to identify medication burden. In the right setting and at the right time, deprescribing provides a real opportunity to minimise side effects and unwanted interactions. From an NHS perspective, deprescribing also has the potential to save costs and it may be seen as such from a patient perspective. This issue merits further consideration but falls outside the scope of this article. Deprescribing can constitute a withdrawal of medication or a dose reduction. Different models and tools exist to aid decisions around safe deprescribing. Individualised tailored review, working with patients, will support improved adherence and thus effectiveness of remaining medication. Conversations with patients may raise issues of cost as a driver for deprescribing However, the question remains-where does the prescriber stand if they stop a medication for which there is an evidencebased guideline to use it?

\section{LITIGATION}

Before considering potential for litigation in deprescribing, we first outline the concepts of clinical negligence and consent in a general context.

\section{Clinical negligence}

A healthcare professional is open to a claim of clinical negligence if their actions fall below the reasonable standard of their peers. (This article uses the term clinical negligence to imply negligence on the part of any healthcare professional, including doctors, pharmacists, prescribing pharmacists and prescribing nurses).

To succeed in a claim of clinical negligence, a claimant must establish all of the elements in box 1 .

\section{Breach of duty}

In order to prove that a healthcare professional such as a pharmacist is negligent, it is necessary to establish that no reasonably competent pharmacist, at the relevant time, with the same qualifications and expertise, faced with the same circumstances, would have acted the same way. If it is possible to show that a reasonable and competent body of pharmacist opinion (of the same grade and area of practice) would have acted in the same way, then the care is not considered negligent.

Therefore when examining the decision of a pharmacist and determining whether it was negligent, it will be necessary to consider their grade and practice area. Put simply, a healthcare professional is judged by their peers in that country; a respiratory consultant is compared with reasonable respiratory consultants, a hospital pharmacist is compared with hospital pharmacists and so on. If a patient consults their general practitioner who then fails to detect a tumour or refer for further investigation, one needs to consider whether a reasonable body of general practitioners, faced with the same set of symptoms, would have acted in the same way. That general practitioner is not expected to meet the standards of, for example, a consultant oncologist in a tertiary centre.

One is also judged by the standards and information available at that time. Medical knowledge, guidelines and diagnostic tools are constantly developing. The fact that a certain medication was subsequently withdrawn or specific guidelines emerged curtailing use in certain cohorts of patients does not mean that a prescriber would be criticised for acting in line with the reasonable body of practitioners at the time. While most if not all pharmacists would be critical of a prescription of diclofenac as a

\section{Box 1 Legal test for clinical negligence}

A duty of care-A healthcare professional has a clear duty of care to patients under their care.

AND

Breach of duty-It must be shown that the claimant did not receive the appropriate standard of reasonable care. This is established where it can be shown that no other reasonable practitioner of like expertise, skill and experience, faced with the same set of circumstances would have acted likewise. In the UK as detailed in the body of this article the test to assess the appropriate standard of reasonable care is known as the Bolam test* (from the case Bolam v Friern Hospital Management Committee. ${ }^{6}$ )

AND

Harm was caused

AND

Causation arises where it can be shown that but for the negligent act or omission, the outcome would have been different, that is, the breach in duty caused the adverse outcome which arose. This link can be difficult to prove.

[*The law governing informed consent is no longer based on the Bolam test. The test has been superseded in relation to informed consent as detailed below. However the Bolam test very much remains as the relevant legal test for other alleged acts of clinical negligence.]

first choice non-steroidal anti-inflammatory drug postoperatively, it was at one time accepted to use this medication routinely post surgery. Reasonable practice is not judged with the benefit of hindsight.

However it must be noted that the mere fact that a professional defends their action by pointing to a reasonable body of opinion is not an automatic defence. The courts can and will consider whether or not the professional standard applied was so defective that it is indefensible. A thorough discussion of this is outside the scope of this article.

\section{Causation}

Causation can be the most difficult hurdle in a clinical negligence action. This links the negligent act with the ultimate unfortunate outcome. It needs to be established that but for the negligent act or omission (ie, error) on the part of that professional, the injury/poor outcome would not have occurred. In brief it must be shown that the breach in duty caused the adverse outcome which arose.

The historic case of Barnett $v$ Chelsea \& Kensington Hospital Management Committee provides a useful example of causation. ${ }^{7}$ A workman became unwell after drinking tea and presented to hospital. Medics turned him away. It transpired that other reasonable practitioners would have admitted him in those circumstances thereby establishing breach of duty on the part of the medics who discharged him. He died shortly afterwards and it transpired that the tea had been poisoned with arsenic. The man's widow sued the hospital for negligently discharging him but lost the case. The defendant's expert evidence succeeded in establishing that, given the short time which elapsed between when the deceased ingested the arsenic and his presentation to hospital, he was in fact going to die anyway. The fact that he was discharged had no bearing on the ultimate 
outcome. One could not say that but for the hospital's action to discharge him, he would have survived.

\section{Consent}

Apart from, or in addition to, a claim in clinical negligence, a healthcare professional is open to a claim based on failure to obtain appropriate informed consent. Recent case law has changed the rules and obligations in relation to informed consent in the UK essentially making it easier for a claimant to succeed in a case based on lack of informed consent.

Any medical procedure or intervention requires consent. (In the absence of consent, practitioners can rely on exceptions such as the doctrine of necessity, eg, in emergency medicine). To be valid consent requires three essential components-it must be free, full and informed-that is, a patient must have the capacity to make the decision in full knowledge of all relevant information and must do so voluntarily. ${ }^{8}$

\section{What is required for consent to be informed?}

The law in the UK has recently been examined and clarified this concept. In March 2015, a seven-judge UK Supreme Court handed down the landmark decision of Montgomery $v$ Lanarkshire Health Board. ${ }^{9}$ The Court unanimously decided that to satisfy the criteria of informed consent, patients must be made aware of any material risks of a proposed intervention, however small that risk may be, and be made aware of any reasonable alternative or variant treatments. This was endorsed shortly afterwards in the case of Spencer $v$ Hillingdon Hospital NHS Trust ${ }^{10}$ which expanded the concept to a postoperative setting.

The focus is now on the particular patient and what that individual 'ordinary sensible patient' in their position would want to know. The information must be explained in a way that the patient understands. Practitioners can no longer hide behind 'prescriber knows best' or provide a selective generalised disclosure of what a reasonable practitioner thinks a patient should be told. If a patient is not told of a risk, and that risk subsequently materialises, they may well succeed in a case for failure of the practitioner to fully inform them of the options. However the claimant will still have to establish that had they been informed of the risk, that they would have chosen a different option. It is not enough to establish that they had not been told of a certain risk, it must be established that if the particular patient had known of that risk they would have opted for a different treatment or course of action.

To succeed in a claim of lack of informed consent, a claimant must establish all of the elements in box 2 .

This patient-centred law enshrines what was already explicit in the General Medical Council's guidance on consent. ${ }^{11}$ The Supreme Court decision in Montgomery confirms this. Updated pharmacist prescribing guidelines are imminent and it is expected that they will mirror the General Medical Council guidelines. Current General Pharmaceutical Council guidelines on consent ${ }^{12}$ include details of requirements for valid consent, appropriate information provision to the patient and withdrawal of consent.

In summary, a practitioner can be open to a claim based on two distinct areas of the law as summarised in box 3. In practice it is likely that the two claims would go hand in hand as part of any proceedings taken.

\section{DEPRESCRIBING AND LEGAL IMPLICATIONS}

Legally, deprescribing has the same status as prescribing: both require use of clinical expertise to carefully review medication

\section{Box 2 Legal test for informed consent}

Lack of informed consent obtained for procedure/treatmentThe healthcare professional failed to take reasonable care to ensure that the particular patient is aware of any material risks involved in any recommended treatment, and of any reasonable alternative or variant treatments.

AND

Harm was caused - that risk, which was not outlined, materialised.

AND

Causation arises where the claimant can establish that had they been informed of the risk which materialised that they would have chosen a different option.

and recommend that a medication be commenced, reduced or withdrawn for patient benefit.

A prescriber is open to a claim of clinical negligence if they do not prescribe appropriately in line with their peers. A prescriber is equally open to a claim of clinical negligence around deprescribing if it is deemed that a similar prescriber acting reasonably would have reviewed a medication and outlined to the patient why they recommend it be reduced or withdrawn and this was not done.

A prescriber must obtain informed consent and advise a patient of potential risks of a medication when prescribing, continuing or increasing a medication. This consent may be documented but more commonly consent is considered implicit if the patient accepts a prescription for a medication. Similarly potential risks on withdrawal or reduction must be outlined. As regards consent, again, legally deprescribing is no different to prescribing: there is a risk associated with both deprescribing and not deprescribing (ie, continuing to prescribe). A patient is entitled to a full disclosure of all material risks whether it is on starting or stopping the medication. Consideration of managing adverse irreversible consequences, part of the initial discussion for deprescribing, will contribute to the decision to deprescribe. Prescribers should explore with the patient risks or potential problems from stopping medication including common problems they might expect, in addition to those of a potential serious nature and those that that individual patient is likely to attach significance to. ${ }^{13} 14$

It is important that the discussion of risks be tailored to the individual patient. An Australian High Court case of Rogers $v$ Whitaker $^{15}$ in the 1990 s, is still relevant and illustrates the issue

\section{Box 3 Potential clinical negligence legal actions}

A claim based on clinical negligence, where the practitioner has failed to act in the manner of a reasonable, similarly qualified practitioner which caused the ultimate adverse outcome. AND/OR

A claim based on the failure to obtain appropriate informed consent of the patient, where if the patient had been made aware of certain material risks of the treatment such information would have caused the patient to opt for a different course of treatment. 
of 'a material risk'. In this case, eye surgery was proposed. The ophthalmic surgeon did not inform the patient Ms Whitaker that the surgery carried a 1 in 14000 risk of blindness in one eye. However this patient was already blind in the other eye, making the remote risk of blindness very 'material' to the patient Ms Whitaker. The patient developed blindness and the Court found the doctor's failure to disclose the risk in this instance to be negligent.

We must also be mindful that what is material to one patient may not be to another. A risk of increased falls on continuing a diuretic may be very relevant to an elderly patient living alone and in that instance it would be important to discuss the risk of not deprescribing. A risk of teratogenic effects of an antiepileptic medication may be of utmost importance to a young female patient in a consideration of whether to deprescribe whereas an elderly male driver may place significantly more emphasis on the risk of a seizure reoccurrence. What constitutes a material risk is a matter of professional judgement, tailored to each individual patient and this begs the question 'How do we determine what a patient considers to be a material risk?'

One might suggest that on starting and stopping a medication, the prudent prescriber would be required to disclose all general side effects and risks but beyond that it is a vague test of what that patient may deem material. The prudent prescriber will equally apply the consultation skills, such as described in the Centre for Postgraduate Pharmacy Education document, ${ }^{16}$ to the practice of prescribing and deprescribing. The Centre for Pharmacy Postgraduate Education (CPPE) advocates the use of a health coaching approach in the context of deprescribing to raise patient awareness of the issues around stopping medicines and promote shared decision making. We suggest that patient-centred consultations, such as seen in this approach, are key to supporting safe deprescribing practice.

The content of a prescribing consultation is described in general terms by the Royal Pharmaceutical Society (RPS) for community pharmacists in the suggested medicines charter for patients ${ }^{17}$ and in the RPS hospital standards ${ }^{18}$ for hospital practitioners. However guidance varies according to the medication prescribed and the individual patient and this is equally true for deprescribing consultations. It is therefore important that prescribers focus on the patient's deprescribing agenda and ask questions, such as, 'in terms of stopping this medicine, what would you like to discuss?' or 'what concerns you about continuing/stopping your medicine?' The challenge lies in the paucity of evidence-based guidance for deprescribing and reinforces the need for a tailored approach according to clinical situation and patient context.

As deprescribing gathers momentum and in time becomes routine, prescribers who fail to undertake regular medication review and consider deprescribing will be acting outside the 'norm' of a reasonable body of practitioners. As patient expectations change to include medication review, prescribers who do not consider deprescribing may be open to a claim of clinical negligence if an adverse outcome arises due to the continued medication.

It remains to be seen how the changes to consent processes will materialise in practice. Arguably, the new rules to provide fuller, more extensive information when making an intervention will result in more open discussions about risks and adverse effects. We hope that will mean that a discussion of risks around deprescribing a medication will facilitate more frequent, appropriate deprescribing. Equally as patients become more informed, it will be patients who challenge prescribers not offering medication review and the option of deprescribing.

\section{THE CHALLENGE OF DEPRESCRIBING IN PRACTICE}

A recent paper suggests that factors such as lack of training, awareness and time are cited as barriers to deprescribing. ${ }^{19} \mathrm{We}$ may surmise that the more cautious among us attribute the reluctance to deprescribe as a combination of fear of adverse clinical sequelae and/or legal repercussions.

Adverse clinical effects can occur with any intervention, prescribing or deprescribing. It is our role to apply clinical knowledge, experience and resources to pre-empt and minimise these adverse outcomes. If a recommendation is based on a clinical assessment of the specific patient and their specific needs, justifiable to a reasonable body of practitioners, a claim of clinical negligence is unlikely to succeed. Decisions made with patients, following full disclosure of all material risks, will minimise the risk of claims for lack of informed consent.

Numerous tools and aids are available to support safe deprescribing, for example the STOPP/START tool, Med stopper online program, use of the medicines appropriateness index, anticholinergic burden, and review of drugs and falls risk. ${ }^{20-24}$ although validation and robustness vary. Conversations with patients and/or their carers/relatives about the risks and benefits from the clinician and patient perspective are crucial to safe deprescribing. A valuable example of such work can be seen on the UK Health foundation website ${ }^{25}$ which demonstrates a patient-centred deprescribing process similar to that described in the literature. ${ }^{26}$

Deprescribing must be undertaken in the context of overall medication review and any decision to prescribe or deprescribe can be reviewed and/or reversed. Decision making should be individualised to the patient, dynamic and ongoing in the quest for optimal patient care. In time it is hoped that more guidelines will incorporate guidance regarding deprescribing and enable evidence-based deprescribing to become commonplace.

\section{SUMMARY}

Legally deprescribing is no different to prescribing (within the UK's legal system). Ongoing review and monitoring of all decisions based on patient and medication-specific factors is required for safe patient care and all decisions must be informed by full and frank informed patient consent. As deprescribing becomes the norm practitioners who fail to consider deprescribing and fail to advise patients of the potential benefits and options may expose themselves to clinical negligence claims. Patient consent to stop, start, change or reduce a medicine must be based on full disclosure of all material risks to that patient. In support of the 'ordinary sensible patient' we encourage all prescribers to consider discussing the risks and benefits of

\section{What this paper adds}

What is already known on this subject?

- Deprescribing by clinicians is uncommon in clinical practice.

- Problems with prescription of multiple medicines makes deprescribing appropriate for consideration. Pharmacists fear the legal implications of deprescribing.

What this study adds?

- There is little evidence-based guidance to support clinicians with deprescribing.

- There is no barrier to deprescribing in law however it is unclear how new legal developments regarding informed consent would apply to deprescribing. 
continuing a medication and the options for deprescribing at the time of prescribing and throughout the patient journey. In the absence of deprescribing guidance as part of evidence-based guidelines, use of deprescribing tools and models are encouraged to support safe practice and improve clinical outcomes. When deprescribing is undertaken in partnership with patients, supported by the knowledge, skills and experience of both patient and clinicians and the patient's values and preferences based on clinical skill, judgement and evidence-based medicine, the law presents no barriers to deprescribing.

Competing interests None declared.

Provenance and peer review Not commissioned; externally peer reviewed.

\section{REFERENCES}

1 Smith R, Rennie D. Evidence based medicine-an oral history. BMJ 2014;348: g371.

2 Cochrane Library. Cochrane Community Archive. http://community.cochrane.org/ about-us/history) (accessed 1 Mar 2016).

3 National Institute of Clinical Excellence (https://www.nice.org.uk/about/who-we-are) (accessed 1 Mar 2016).

4 Ward C. Unpublished audit of number of medicines of older inpatient and outpatients at Northwick Park Hospital, Harrow, UK, 1981.

5 Mulhem E, Lick D, Varughese J, et al. Adherence to medications after hospital discharge in the elderly. Int J Family Med 2013;2013:901845.

6 (1957) 1 WLR 583.

7 [1969] 1 Queen's Bench 428.

8 Mental Capacity Act 2005 http://www.legislation.gov.uk/ukpga/2005/9/contents. (accessed Feb 2016).

9 [2015] UKSC 11

10 [2015] EWHC 1058 (QB).

11 General Medical Council. Guidance on consent http://www.gmc-uk.org/guidance/ ethical_guidance/14316.asp (accessed Feb 2016).
12 General Pharmaceutical Council. Guidance on consent February 2012. https:// www.pharmacyregulation.org/sites/default/files/guidance_on_consent_08. 09.14_0.pdf

13 Sokol DK. Let's raise a glass to the ordinary sensible patient. BMJ 2015;351:h3956

14 Sokol D. Update on the UK law on consent. BMJ 2015;350:h1481.

15 (1992) 175 CLR 479.

16 Grimes L Barnett N. Section 5. Health coaching in patient consultations. Consultation skills for pharmacy taking a patient centred approach. Feb 2014 Centre for pharmacy postgraduate education http://www.consultationskillsfor pharmacy.com/docs/docb.pdf

17 Royal Pharmaeutical Society. Medicines Charter for patients: What you should expect from your community pharmacist. https://www.rpharms.com/ news-story-downloads/consultation---medicines-charter-for-patients.pdf

18 Royal Pharmaceutical Society. Professional Standards for Hospital Pharmacy Services Optimising Patient Outcomes from Medicines July 2014. http://www.rpharms.com/ support-pdfs/rps — professional-standards-for-hospital-pharmacy.pdf (accessed 1 Mar 2016).

19 Jubraj B, Marvin V, Poots AJ, et al. A pilot survey of junior doctors' attitudes and awareness around medication review: time to change our educational approach? Eur J Hosp Pharm 2015;22:243-8.

20 O'Mahoney D, O'Sullivan D, Byrne S, et al. STOPP/START criteria for potentially inappropriate prescribing in older people: version 2. Age Ageing 2014;44:213-18

21 McKormack J, Mangin D, Farrell B, et al. Medstopper tool. http://medstopper.com/ (accessed 19 May 2016).

22 Hanlon JT, Schmader KE, Samsa GP, et al. A method for assessing drug therapy appropriateness. J Clin Epidemiol 1992;45:1045-51.

23 Salahudeen MS, Duffull SB, Nishtala PS. Anticholinergic burden quantified by anticholinergic risk scales and adverse outcomes in older people: a systematic review. BMC Geriatr 2015;15:31.

24 De Jong MR, Van Der Elst M, Hartholt KA. Drug-related falls in older patients: implicated drugs, consequences, and possible prevention strategies. Ther Adv Drug Saf 2013:4:147-54.

25 The Health Foundation. Pills: reviewing medication in care homes. http://www. health.org.uk/pills (accessed 1 Mar 2016).

26 Barnett N, Oboh L, Smith K, et al. Patient-centred management of polypharmacy: a process for practice. Eur J Hosp Pharm 2016;23:113-17. 